Physics

Physics Research Publications

\title{
Modeling Gaussian-to-self-avoiding-walk crossover for stiff chain polymers
}
M. F. Islam
H. Nakanishi 


\title{
Modeling Gaussian-to-self-avoiding-walk crossover for stiff chain polymers
}

\author{
Md. Fhokrul Islam and Hisao Nakanishi \\ Department of Physics, Purdue University, West Lafayette, Indiana 47907, USA
}

(Received 1 June 2006; published 6 November 2006)

\begin{abstract}
We explore the long-standing discrepancy between an experimentally observed sudden onset of the excluded-volume effect in stiff chains [Murakami et al., Macromolecules 13, 345 (1980)] and much more gradual onset seen by Monte Carlo simulations of the persistent self-avoiding walk model. We find by using various simulations and real-space renormalization methods that, while models incorporating parallel-bond inhibition enhance the excluded-volume effect, they have little effect on the abruptness of its onset. We also observe that relaxational simulations, which include reptationlike motion can, however, lead to a sudden onset much like in the experimental observation.
\end{abstract}

DOI: 10.1103/PhysRevE.74.051107

PACS number(s): 05.50.+q, 61.41.+e, 64.60.Fr, 05.40.Fb

\section{INTRODUCTION}

Some years ago, Murakami et al. [1] reported the result of light scattering and other measurements of an extremely stiff linear polymer, poly-hexyl-isocyanate (PHIC) with narrow molecular weight dispersion in hexane solvent at room temperature. They analyzed the dependence of the radius of gyration on the molecular weight and reported a rather sudden departure from the standard Kratky-Porod wormlike chain model [2] as shown in Fig. 1. To complicate the matter further, viscosity measurements did not show a corresponding abruptness as the molecular weight increased. However, we have not been able to find any other similar experiments in the literature and thus these results appear to be uncorroborated by other experiments so far. Moon and Nakanishi [3] subsequently performed simulations of a stiff self-avoiding walk model called the persistent self-avoiding walk (PSAW) on three-dimensional lattices and showed that the stiffness alone cannot explain the sudden increase in the radius of gyration as observed by Murakami et al. Their analysis of Murakami et al.'s radius of gyration data suggested that the stiff polymer behaved as if there were no excluded-volume effects up to a certain molecular weight (or length) and then, beyond this point, the excluded-volume effect sets in abruptly, making the chain behave almost like a flexible selfavoiding walk. The discrepancy between the PSAW model and experimental results certainly calls for new work on both theoretical and experimental fronts.

In this work, we explore this discrepancy theoretically and numerically by crudely modeling some of the obvious differences between the stiff PHIC and the PSAW. PHIC has large pendant side chains, which are individually almost flat. Large side groups clearly increase excluded volume and, while it can enhance the stiffness of the chain, it could also compete with it. Such competition would occur, e.g., if the side groups from different parts of the chain interfere in a trans configuration (straight path) and thus prefer a gauche configuration (a turn) instead. Of course, it is unclear whether such competition is beyond the simple effects of larger excluded volume overall. Nonetheless, an inclusion of effects that directly compete with the stiffness appears intriguing. The model we consider first (model 1) is a lattice self-avoiding walk with the monomer length equal to a lat- tice constant and the side chain length equal to half a lattice constant. We then assign a short-range repulsive interaction $\epsilon_{1}>0$ and an on-site repulsive interaction $\epsilon_{2}>0$ to the side chains. A square-lattice version is illustrated in Fig. 2. The repulsion $\epsilon_{1}$ induces stiffness while the soft-core on-site repulsion $\epsilon_{2}$ may favor a gauche turn as in the example shown. In this way, the model contains some competition between stiffness and excluded-volume of the side chains.

On the square lattice, model 1 is almost equivalent to simply giving energy $\epsilon_{1}$ for each gauche step of the backbone and $\epsilon_{2}$ for each nearest-neighbor, parallel bond pair. Though the model illustrated in Fig. 2 has a more direct physical interpretation, we will use the latter model (denoted by model 2) in what follows for simplicity. We extend this latter model to three dimensions by simply assigning $\epsilon_{1}$ to each gauche turn and $\epsilon_{2}$ to each neighboring pair of parallel bonds (without explicitly invoking side chains). So the canonical model we start with is given by the effective Hamiltonian for a self-avoiding chain of the form

$$
H=N_{G} \epsilon_{1}+N_{P B} \epsilon_{2} \text { (model 2), }
$$

where $N_{G}$ is the number of turns and $N_{P B}$ is the number of neighboring parallel bond pairs. Moreover, in much of this

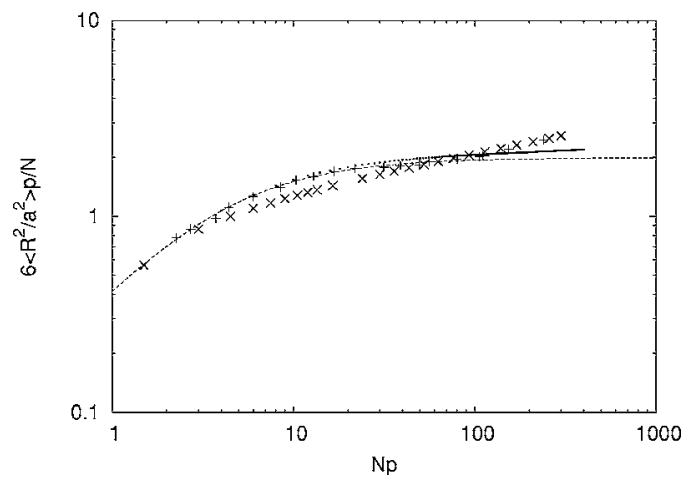

FIG. 1. The PHIC radius of gyration results of Murakami et al. [1] (pluses) are compared with the Gaussian, stiff chain prediction (dashed line), PSAW Monte Carlo results of Moon and Nakanishi [3] (dots), and (almost) flexible SAW results (crosses). $N$ is the number of steps (monomers) and $p$ is the gauche probability. This is a scaling plot in that the abscissa is the number of persistence length segments $(N p)$ and the ordinate is the scaled squared radius of gyration appropriate in the stiff regime $(N \rightarrow \infty, p \rightarrow 0)$. 


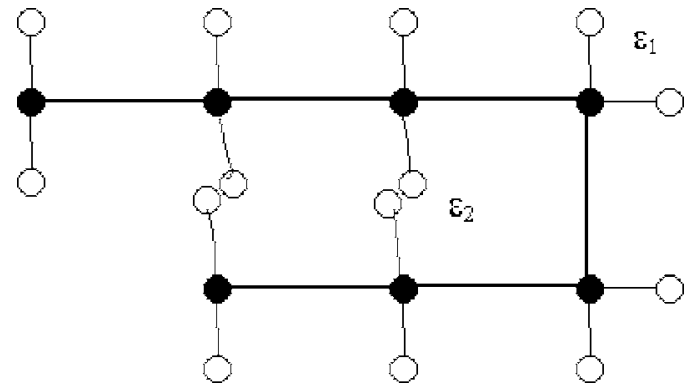

FIG. 2. A sketch of the model 1 of a stiff chain with repulsive side groups. The side chains have both a short-ranged repulsion and an on-site repulsion. If two successive gauche turns happen to occur, the next step along the chain may either satisfy $\epsilon_{1}$ and go trans or satisfy $\epsilon_{2}$ and go gauche.

work, we present results from setting $\epsilon_{2}$ to be large, i.e., essentially forbidding neighboring parallel bonds as the limiting case of (relatively) large competition against the stiffness.

\section{MODIFIED PSAW MODEL}

In the PSAW model of a stiff chain, each step of a chain is randomly and independently generated with a given gauche probability, where any attempt at self-intersection results in the rejection of the walk from the ensemble. The gauche probability $p$ corresponds to the energy $\epsilon_{1}$ by

$$
p=\frac{z-2}{z-2+\exp \left(E_{c}\right)},
$$

where $E_{c} \equiv \epsilon_{1} / k_{B} T$ and the lattice coordination number $z=6$ for the simple cubic lattice. This correspondence ensures the correct relative statistical weights of trans and gauche steps if there is no other competing interaction, or $\epsilon_{2}=0$. It is also true if $\epsilon_{2} \rightarrow \infty$, or all walks with nearest-neighbor parallel bond pairs are rejected from the ensemble. However, this is no longer true if $\epsilon_{2}$ is finite. As it is difficult to take a finite $\epsilon_{2}>0$ into account in such a random walk model, we consider a modified PSAW simulation for the limit of $\epsilon_{2} \rightarrow \infty$ (which we label as model 3). This can be incorporated into a modified PSAW by generating random realizations of a PSAW of the gauche probability equal to Eq. (2) and rejecting them whenever prohibited nearest-neighbor, parallel bond pairs appear in the chain.

Shown in Fig. 3 is the radius of gyration $\left\langle R_{g}^{2}\right\rangle$ for the modified PSAW (with the parallel-bond inhibition), standard PSAW, and the Gaussian stiff chain result (wormlike chain) (we call the last model persistent random walk or PRW) in terms of the wormlike-chain scaling variables. The two PSAW results are from a fixed stiffness of $p=0.1$ and only the number of steps $N$ has been varied. (The corresponding data from $p=0.05$ are almost identical.) Clearly, the behavior of the modified PSAW is quite similar to that of the standard PSAW. While it shows a larger deviation from the PRW result (thus larger excluded volume in general), there is evidently no indication of sharpening in the onset of the excluded volume effect. Analogous results for smaller $p$ are

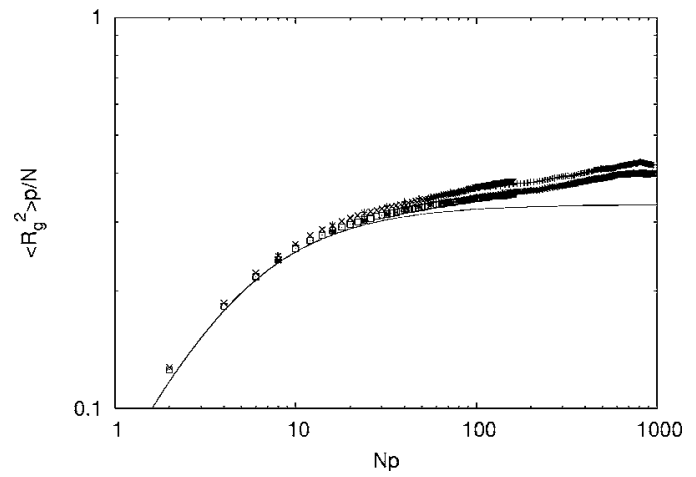

FIG. 3. The scaled, square radius of gyration from the modified PSAW model with nearest-neighbor parallel bonds prohibited $(\times$ and + ) compared with those of the original PSAW model (squares and $*$ ) and the Gaussian stiff chain (or PRW) result (solid line).

also consistent: there is no change in the way excluded volume effects set in. In fact, smaller $p$ shifts the onset of the flexible SAW behavior even further toward larger $N$ and thus all curves appear even closer to each other.

These PSAW simulation results were obtained from at least 10000 realizations (with and without enrichment techniques), and the standard error of the mean was smaller than the symbols used in the figures. Further, the realizations were divided into several batches and their means and fluctuations are compared and found to be quite comparable to each other. Also, this type of simulation, where PSAWs are grown from a seed until self-intersection is attempted and upon such an attempt a whole new PSAW is started, is more likely to be able to sample rare but important configurations than some other types of simulations (cf. Sec. IV). Thus the present PSAW results appear to be close to equilibrium. Therefore, we interpret these results as suggesting that, at least in equilibrium, the parallel-bond inhibition of model 3 only increases the overall excluded-volume effect but does not lead to a sudden onset of such effects.

\section{REAL-SPACE RENORMALIZATION}

In an attempt to corroborate the simulations of model 3, we extended the real-space renormalization methods of Lee and Nakanishi [4] who applied the technique to the standard PSAW model in two and three dimensions. They obtained results that are consistent with successive crossovers from a stiff rod to Gaussian stiff chain and finally to an excludedvolume stiff chain regime in three dimensions but the corresponding results in two dimensions showed a direct crossover from a stiff rod to the excluded volume stiff chain regime. This was consistent with both the theoretical expectations [5] and the PSAW Monte Carlo results referred to earlier. However, it did not address the crossover abruptness nor other possible effects competing with stiffness. While it is possible to include cases corresponding to finite $\epsilon_{2}$ of model 2 by assigning a fugacity for the occurrences of neighboring parallel-bond pairs and including this fugacity in renormalization, we opted to address only the $\epsilon_{2} \rightarrow \infty$ limit or the model 3 in this work. We also did not include a parameter 


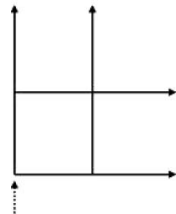

(a)

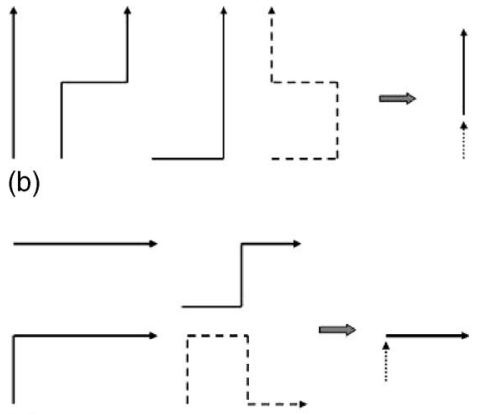

(c)
FIG. 4. (a) $2 \times 2$ renormalization cell into whose corner a PSAW enters from below; (b) the PSAW configurations that renormalize to a trans superstep. Neighboring parallel bonds occur in the dashed configuration and are excluded from $K^{\prime}=2 K G^{2}+K^{2}$. (c) Those that renormalize to a gauche superstep. The dashed configuration contains a neighboring parallel-bond pair and is excluded from $G^{\prime}=G^{3}+K G+K^{2} G$.

that would control the degree of excluded volume, or in other words, the chains were always constrained to be fully selfavoiding. Thus we keep the simple, two-parameter framework and only aim to look for the possible effect of prohibiting neighboring parallel bonds on the manner of stiff-toflexible crossover.

To include the parallel-bond inhibition of model 3, we simply exclude those configurations that contain any pairs of neighboring parallel bonds within the sums that contribute to renormalized trans and gauche bond fugacities. Thus, after Lee and Nakanishi [4], we denote the trans bond fugacity by $K$ and the gauche bond fugacity by $G$, and write the recursion relations for them as

$$
\begin{aligned}
K^{\prime} & =\sum_{\text {trans }} K^{N_{T}} G^{N_{G},}, \\
G^{\prime} & =\sum_{\text {gauche }} K^{N_{T}} G^{N_{G}},
\end{aligned}
$$

where $\Sigma_{\text {trans }}$ denotes the sum over all configurations of PSAW's that enter from a corner of a cell and exit it in the same direction while $\Sigma_{\text {gauche }}$ denotes those that exit in a different direction. $N_{T}$ and $N_{G}$ refer to the number of trans and gauche bonds, respectively, for each configuration in the sum, and in our application, the sums exclude the configurations with neighboring parallel bonds. Figure 4 shows this for a $2 \times 2$ cell on the square lattice for illustration purposes only (as our interest is in three dimensions).

For $2 \times 2 \times 2$ cells on the simple cubic lattice, these equations are

$$
\begin{aligned}
& K^{\prime}=2 G^{4}+4 G^{5}+2 G^{6}+K\left(4 G^{2}+4 G^{3}\right)+K^{2} \\
G^{\prime}= & 2 G^{3}+3 G^{4}+2 G^{5}+G^{6}+K\left(G+G^{2}+2 G^{3}+2 G^{4}+G^{5}\right) \\
+ & K^{2}\left(G+G^{2}\right) .
\end{aligned}
$$

For $3 \times 3 \times 3$ and larger cells, the recursion relations are too lengthy to quote here. (For example, for $3 \times 3 \times 3$, there are up to 10 trans and 20 gauche bonds that appear in the recur-

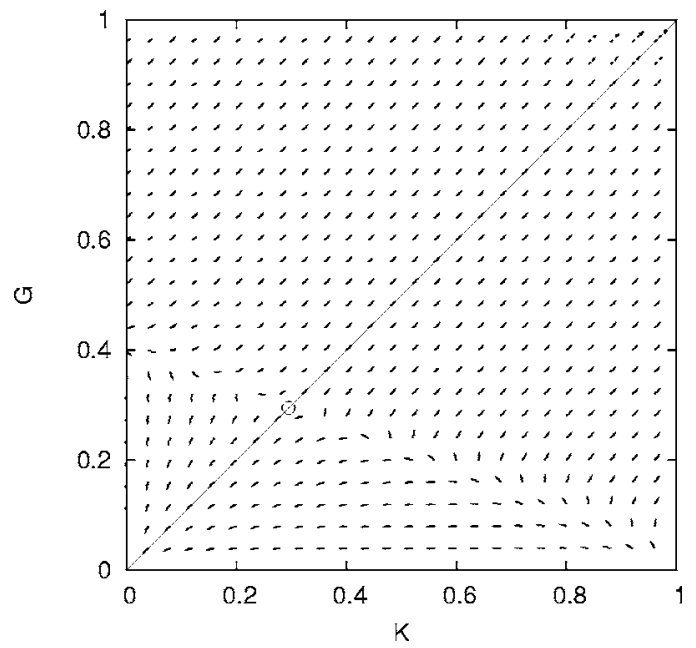

FIG. 5. Renormalization flows generated by the recursion relations for the $4 \times 4 \times 4$ PSAW with parallel-bond inhibition. The arrows indicate the local direction of the flows from $(K, G)$ to $\left(K^{\prime}, G^{\prime}\right)$. Also shown is the symmetry axis $K=G$ and the critical fixed point on it at $K^{*}=G^{*}=0.2952$ (a small circle).

sion relations with coefficients of some of the terms of greater than 700 , and for $4 \times 4 \times 4$, there are up to 23 trans and 50 gauche bonds where the maximum coefficients have values greater than 490 million.)

Shown in Fig. 5 is the resulting renormalization flow diagram from the $4 \times 4 \times 4$ cell calculation. It turns out that the general features of this diagram are rather similar to the corresponding flow diagrams of the PSAW renormalization without parallel-bond inhibition. They both show a critical fixed point on the symmetry axis $K=G$ (i.e., a flexible chain) that is stable to stiffness (i.e., in the direction perpendicular to $K=G$ ), and there is no apparent qualitative difference between this picture and that of the ordinary PSAW (though we performed the latter only up to $3 \times 3 \times 3$ ). The only differences are quantitative, and here we compare for only the $3 \times 3 \times 3$ results since we have done both problems only up to this size: The fixed-point value of $K^{*}=0.3067$ (for $3 \times 3 \times 3$ ) is about $11 \%$ larger than that of the usual PSAW and the relevant eigenvalue $\Lambda_{1}=5.66$ (in the $K=G$ direction) is about $14 \%$ smaller while the irrelevant eigenvalue $\Lambda_{2}=0.188$ (in the stiffness direction) is about $29 \%$ larger than the respective, corresponding values for the usual PSAW renormalization of the same cell size.

Since $K^{*}$ is an estimate of the $1 / \mu$, where $\mu$ is the effective coordination number, the larger value of $K^{*}$ implies that on average a smaller number of turning configurations are available, which is consistent with a larger excluded-volume effect. The smaller $\Lambda_{1}$ could imply a larger estimate of the Flory exponent $\nu$, but such an estimate from a single, small cell calculation is not significant in itself; we would need to study the trends as the cell sizes are varied. The ratio $\phi \equiv\left|\ln \Lambda_{2} / \ln \Lambda_{1}\right|$ is the correction-to-scaling exponent that measures the relative strength of the deviation from asymptotic power law $\left\langle R^{2}\right\rangle \sim N^{v}$ (the smaller $\phi$, the larger the correction). Here $\phi=0.964$ compared with the corresponding value of 1.02 for the usual PSAW, suggesting only a minor increase in the importance of the correction. 


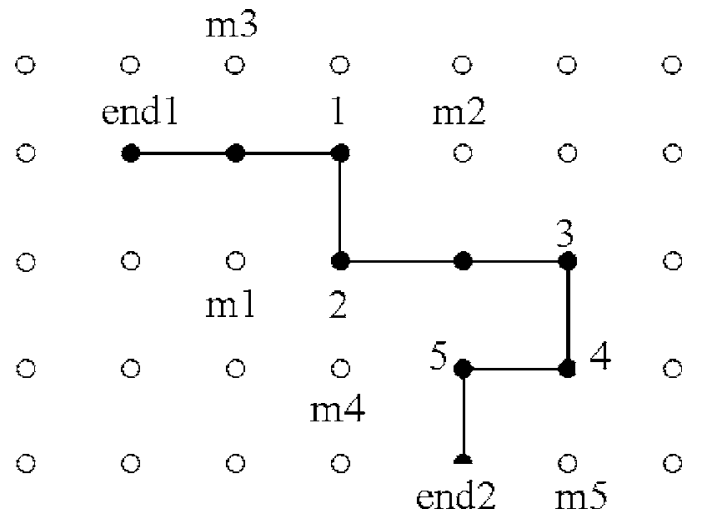

FIG. 6. Illustration of allowed local moves in the relaxational Monte Carlo simulation. The end labeled end 1 can move to either site $\mathrm{m} 1$ or $\mathrm{m} 3$, while the end, end 2 can only move to $\mathrm{m} 4$. The corners 1,2 , and 5 can move to $\mathrm{m} 1, \mathrm{~m} 2$, and $\mathrm{m} 5$, respectively.

Thus, the real-space renormalization we performed does not appear to indicate a significant effect of this particular kind of competition to stiffness (inhibition of parallel bonds) that was included. (However, there may be significant effects in two dimensions [6].) These results are entirely consistent with simply an increased excluded-volume effect, and thus also consistent with the equilibrium Monte Carlo simulation of the previous section.

\section{RELAXATIONAL SIMULATION}

So far, all the indications are that, in equilibrium, prohibiting nearest-neighbor parallel bonds does not affect the manner of onset of the excluded-volume effect in the PSAW model. In an effort to explore this issue further, we now return to model 2 [Eq. (1)] and investigate it by using a relaxational Monte Carlo simulation. In this approach, we can vary the value of $\epsilon_{2}$ as well as track the relaxation of the chain conformation as a function of the Monte Carlo steps. Although the dynamics introduced by our choice of the relaxational pathways may not necessarily accurately reflect the dynamics of a stiff polymer in solution, it at least provides some insight into possible nonequilibrium effects (as we shall see below).

First, we must generate initial configurations of the stiff, self-avoiding chains. We do this by using the original (stochastic) PSAW model with a small gauche probability $p$, typically obtained by using Eq. (2) for the given $\epsilon_{1}$ (but with no regard to $\epsilon_{2}$ ). Next, we must define the elementary steps allowed in the relaxation. As usual in lattice polymer simulations, we first include the local motion of the either end point into an unoccupied lattice site (e.g., end1 in Fig. 6 can move to sites labeled $\mathrm{m} 1$ or $\mathrm{m} 3$ ). Then, corners could flip if this action does not result in self-intersection, as in the figure where the corner 1 can flip to $\mathrm{m} 1$, or corner 2 can flip to $\mathrm{m} 2$, etc. With these two types of local moves allowed, there are six possible moves available to the chain shown in the figure.

It is well known that, for flexible chains $\left(\epsilon_{1}=\epsilon_{2}=0\right)$, the above two kinds of moves are sufficient to quickly equilibrate the chain conformation. However, for stiff chains (large

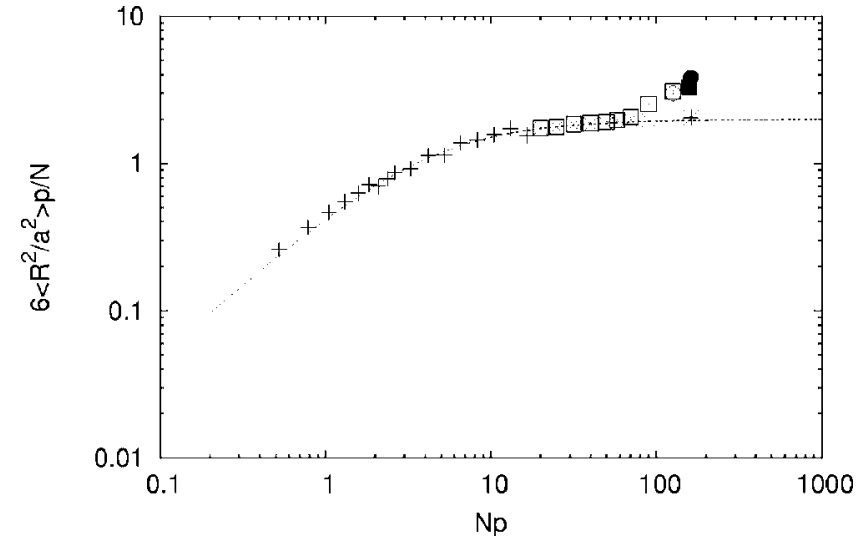

FIG. 7. Some of the results from the reptation relaxational Monte Carlo (symbols) are shown together with the Gaussian PRW results (dashed line). The different symbols correspond to different stiffness (corresponding to the range of stiffness between $p \approx 0.026$ and 0.18 ). Each data point corresponds to the (time) average from at least a few million attempted moves, with those for larger values of $N p$ averaged over 13 million moves or more. The last few points were an average of several independent chains (typically four), while most other points are the averages of many more independent chains.

$\epsilon_{1}>0$, with or without $\epsilon_{2}$ ), the relaxation process is extremely slow and freezes quickly because of the high barrier of introducing gauche turns. To work around this problem, we additionally allow a new type of move called slide. There are two possible kinds of slide moves; an end point can slide outward, away from the current position of the chain, either creating a trans step in its immediate wake or a gauche step. The rest of the monomers will follow the end point and slide accordingly like a snake. With these types of basic moves allowed, the usual Metropolis algorithm is applied and the relaxation process is continued for a predefined number of time steps (defined here as the number of attempted moves).

The advantage of the slide move is obvious as it can circumvent barriers and easily remove existing gauche steps. If, e.g., the chain of Fig. 3 slides one step to its left, the number of corners in the chain is reduced from five to four, thereby reducing the total corner energy, arriving at an energetically favorable configuration. It is also possible to get a larger number of corners as well, though less likely to be accepted according to the Metropolis algorithm. However, as with any set of basic allowed moves, this scheme introduces a particular dynamics, and since it is impossible to make sure to reach statistical equilibrium, our results may reflect the specific dynamics introduced by the slide moves. Of course, this may be a blessing in disguise since sliding as we introduce is similar to reptation, a kind of dynamics that is believed to be important in real polymer solutions, at least in high concentrations. The extreme stiffness in our problem artificially creates a situation that is somewhat analogous to a concentrated solution, where relaxation to equilibrium is difficult only through localized moves.

The results of these simulations are illustrated in Fig. 7, where the same scaling variables as in Figs. 1 and 3 are also used and the data from $\epsilon_{2}=0$ and $\epsilon_{2}>0$ are compared with each other and with the Gaussian PRW results (dashed line). 


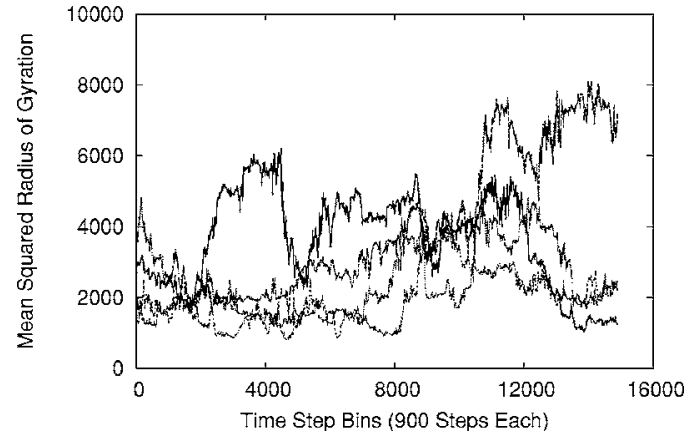

FIG. 8. Typical reptation relaxational Monte Carlo time series are shown for four chains. These results are obtained for a chain of $N=900$ steps simulated for over 13 million attempted moves binned into 15000 points representing averages over 900 moves each.

Most of these data were obtained at $\epsilon_{1}=3.5$, roughly corresponding to a fixed stiffness of $p=0.1$ and for a different number of steps $N$, but some were for different levels of stiffness. Those plotted with,$+ \times$, and $*$ are all for $\epsilon_{2}=0$ (no parallel-bond inhibition), where the stiffness values are $\epsilon_{1}=5(p \approx 0.026), \epsilon_{1}=3.5(p \approx 0.1)$, and $\epsilon_{1}=2.9(p \approx 0.18)$, respectively. Those plotted with $\square, \bigcirc, \boldsymbol{\square}$, and $\bigcirc$ are all with $\epsilon_{2}=20$, i.e., with parallel-bond configurations essentially forbidden, where the stiffness values are $\epsilon_{1}=3.5$ (or $p \approx 0.1$ ), and $\epsilon_{1}=3.2 \quad(p \approx 0.14), \epsilon_{1}=3.27 \quad(p \approx 0.13)$, and $\epsilon_{1}=2.9$ $(p \approx 0.18)$, respectively.

The most striking features are that the last few points for the parallel-bond inhibited case do indeed significantly deviate from the wormlike chain (or Gaussian stiff chain) results while all prior points are almost identical to the Gaussian chain result. Both of these features are rather reminiscent of the PHIC experiment of Murakami et al. in contrast to the PSAW Monte Carlo simulation results with or without parallel-bond inhibition.

Of course, we must take these results with caution. If we regard the PSAW Monte Carlo results as describing equilibrium, as we in fact do, the current reptation Monte Carlo results cannot correspond to equilibrium. Presented in Fig. 8 are the time dependence for four typical, independent reptation runs of the square radius of gyration of a single chain. They correspond to the stiffness of $p \approx 0.1$ and with the parallel-bond configurations essentially prohibited. As can be seen, three of these chains appear to settle into an apparent equilibrium limit at $\left\langle R^{2}\right\rangle \approx 2000$, but one chain has a much wider fluctuation between about 2000 and 7000. Each data point in the figure represents an average over $N=900$ (also the length of the chain) attempted moves, and yet evidently we have not reached a true equilibrium. For reference, a chain that is reptating in a highly concentrated solution or a dense network is thought to require a time that scales as $O\left(N^{3}\right)$ to completely forget its previous conformation [7]. For $N=900$, this so-called tube renewal time alone may amount to more than 700 million steps. Clearly, the current problem of a long, stiff chain is a different problem but it does share some features such as the severe constriction of where the chain can move to. Thus, one possible explanation of the features of Fig. 7 is that the stiff chains (with or without the parallel-bond inhibition) are mostly stuck in a metastable state that is close to the wormlike-chain result, and only some of the parallel-bond inhibited chains make it out of such a state at sufficiently large $N p(\approx 100)$. That is, while the whole range of the results shown in Fig. 7 are nonequilibrium, they may still suggest that the parallel-bond inhibition, in competition with stiffness, does help with the convergence toward a true equilibrium, resulting in the prominent rise in $\left\langle R^{2}\right\rangle$ only for that case for sufficiently long chains. Again, these are speculations, as we do not have resources to run the simulations longer or on more chains to definitively conclude, but all the appearance is that the features referred to above are characteristics associated with dynamically induced metastable behavior, and moreover, competition with stiffness may help to differentiate the dynamical behavior compared to the case only with stiffness.

\section{SUMMARY}

In this work, we attempted to unravel the differences between the one experimental result [1] available for the conformational statistics of very stiff, single polymer chains and those of PSAW lattice simulations [3]. We focused on a particular type of competition to stiffness (parallel-bond inhibition) and used modified PSAW simulation, real-space renormalization, and relaxational, reptation simulation on such a model. The results suggest that such a competition will, in true equilibrium, only lead to an increased excluded-volume effect, but with reptationlike dynamics, the true equilibrium is difficult to obtain and the parallel-bond inhibition could greatly help the chains to get out of the trapped, metastable state if the chain is long enough. Our speculation is that the experimental result [1] may in fact reflect metastable states somewhat like those we observed in the dynamical simulations. With the current findings, further experiments on stiff chain polymers are called for. Such work would shed much light on future avenues of exploration.

\section{ACKNOWLEDGMENTS}

We would like to thank J. Woods Halley for introducing one of us (H.N.) to the problem of stiff chains, and to him, J. Moon, and B. Widom for discussions.
[1] H. Murakami, T. Norisuye, and H. Fujita, Macromolecules 13, 345 (1980).

[2] O. Kratky and G. Porod, Recl. Trav. Chim. Pays-Bas 68, 1106 (1949).

[3] J. Moon and H. Nakanishi, Phys. Rev. A 44, 6427 (1991), and references therein.
[4] S. B. Lee and H. Nakanishi, J. Phys. A 20, L457 (1987).

[5] H. Nakanishi, J. Phys. (Paris) 48, 979 (1987).

[6] H. Nakanishi and F. Islam (unpublished).

[7] P. G. de Gennes, Scaling Concepts in Polymer Physics (Cornell University Press, Ithaca, NY, 1979). 